\title{
Coexistence of Tricubes in Digital Naive Plane
}

\author{
J. Vittone \& J.M. Chassery \\ Laboratoire TIMC-1MAG, Institut Albert Bonniot \\ Domaine de la merci, 38706 La Tronche cedex, France \\ e-mail: \{Joelle.Vittone,Jean-Marc.Chassery\}@imag.fr
}

\begin{abstract}
Tricubes are considered as elementary 3D neighbours used to generate digital planes. We present some properties of these tricubes and discuss about their characterization and coexistence in a digital naive plane.
\end{abstract}

Key words. digital naive plane, bicube, tricube.

\section{Introduction}

Digital naive plane recognition is an important subject of research. Different works on this topic had been developped. An incremental algorithm has been introduced by Debled and Reveillès [DR95][DRR94] and more recently, works have been performed by Schramm based on Fourier's algorithm [FST96]. Here we are interested by the configurations of neighbourhoods, called tricubes, appearing in a digital naive plane. Properties about tricubes are introduced and we propose an array grammar based on tricubes for characterization of naive plane.

\section{Definitions}

A digital naive plane, 18-connected digital plane, is the set of points of $\mathbb{Z}^{3}$ satisfying the double inequality $\mu \leq a x+b y+c z<\mu+\max (|a|,|b|,|c|), a, b, c, \mu \in$ $\mathbb{Z}$, where $(a, b, c)$ is the normal vector of the plane and $\mu$ a translation parameter. Using symetries, we can reduce our study to naive planes such as $0 \leq a \leq b \leq c$. We will note these planes $P(a, b, c, \mu)$.

The lower (resp. upper) leaning points of a naive plane $P(a, b, c, \mu)$ are the points verifying the equation $a x+b y+c z=\mu$ (resp. $a x+b y+c z=$ $\mu+c-1$ ).

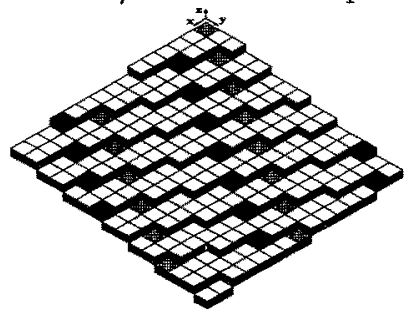

Fig. 1. Naive plane $P(3,7,17,0)$ on $[0,14] \times[0,14]$; in black upper leaning points, in gray lower leaning points. 
A bicube is a set of 4 voxels of a naive plane for which the projection on plane $(0 x y)$ is reduced to a $2 \times 2$ square of pixels (fig.2).

Each voxel $M$ in a naive plane $P$ has 8 neighbours belonging to this plane. We call tricube attached to $M$ in $P$ the set of these 9 voxels. The projection of
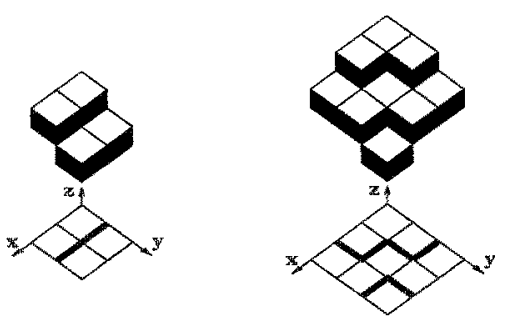
a tricube on the plane $(0 x y)$ is reduced to a $3 \times 3$ square of pixels (fig.2).

Fig. 2. Example of bicubes and tricubes.

\section{Construction of tricubes by grouping bicubes}

There exists 5 different configurations of bicubes (see fig.3) in a naive plane [Fra95][Fra96b][Fra96a].

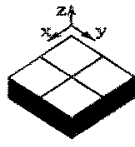

0

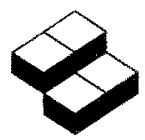

1

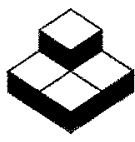

2

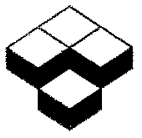

3

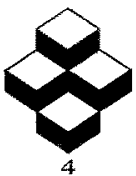

Fig. 3. The 5 different configurations of bicubes.

Bicubes of a naive plane verify the following property:

Property 1. [FST96] A naive plane can't contain simultaneously more than 4 different configurations of bicubes (types 0 and 4 can't appear in a same plane).

A tricube can be seen as a grouping of four adjacent bicubes with recovering. We developped an algorithm to gencrate the set of tricubes. To do that we tested the compatibility of connection between bicubes.

More precisely we used the formal representation of bicubes of figure 4 , where a full segment represents two voxels at same level and dot segment represents two voxels situed at different level.

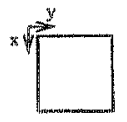

0

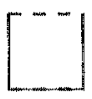

1

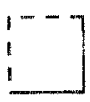

2

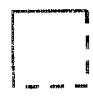

3

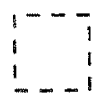

4

Fig. 4. Formal representation of the 5 bicubes. 
When we generate a tricube we note that two bicubes can connect only by a same type of line of voxels.

Now retaining only configurations verifying property 1 , we obtain the 40 existing configurations of tricubes introduced by Debled[DR95] and illustrated in annexe.

\section{Properties of a naive plane through its tricubes}

We recall the following properties from previous works on digital planes:

Property 2. [Rev95] There are no more than 9 different configurations of tricubes in a same naive plane.

Property 3. [Rev95] Each piece of plane which is projected as a $5 \times 5$ square on the plane $(O x y)$ and which is centered on a leaning point contains all the configurations of tricubes appearing in the plane.

Let us introduce the following definitions:

Definition 1. The symetric of a tricube $T$ is the tricube $T^{\prime}$ obtained by symetry around the central voxel of $T$. We notice by [T, T'] symetric tricubes. A tricube $T$ is called neutral if $T=T$ '. We notice by [T] the neutral tricubes. (see example on fig.5)
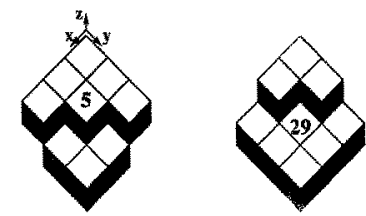

(a)

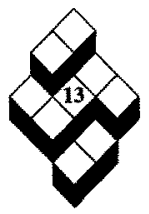

(b)

Fig. 5. Example of: (a) symetric tricubes; (b)a neutral tricube.

and this lemma:

Lemma 1. Let $\left(x_{l}, y_{l}\right)$ be coordinates of the projection on plane $(0 x y)$ of a lower leaning point and $\left(x_{u}, y_{u}\right)$ those of an upper leaning point of a same naive plane. Then for every $(\alpha, \beta) \in Z^{2}$, the configuration of tricube at point $\left(x_{l}+\alpha, y_{l}+\beta\right)$ is symetric from the configuration at point $\left(x_{u_{u}}-\alpha, y_{u}-\beta\right)$.

we have finally:

Proposition 1. If the number of configurations appearing in a naive plane is:

- odd then configurations of tricubes contain pairs of symetric tricubes,

- even then one configuration is neutral and the others are pairs of symetric tricubes. 


\section{Grammar of tricubes}

In order to enumerate digital planes we propose to use the number of tricubes involved in the decomposition of each plane. We have noticed that it exists two types of tricubes: the neutrals and the symetrics. In refering with annexe 1 , the 6 neutral tricubes are numbered by [0], [8], [39], [19], [13] and [32]. We have seen that if a plane is generated with only one configuration of tricubes then this one corresponds to a neutral. Only [0], [8] and [39] can be used to generate a plane. These tricubes generate respectively the planes with normal vector $(0,0,1),(0,1,1)$ and $(1,1,1)$. These planes define the limit of the part of space containing planes such as their normal vector $(a, b, c)$ verify $0 \leq a \leq b \leq c$. So we will use them as a base on which we will construct other configurations.

Each normal vector $(a, b, c)$ with $a, b, c \in \mathbb{Z}$ and $0 \leq a \leq b \leq c$ can be decomposed as $(c-b)(0,0,1)+(b-a)(0,1,1)+a(1,1,1)$. To generate all normal vectors of planes, we introduce a tree such as each node has three sons. At the top we start with vector $(0,0,0)$. Then we generate the three sons of a node $(x, y, z)$ in the following way:

- the left son is the vector $(x, y, z+1)$,

- the middle son is the vector $(x, y+1, z+1)$

- the right son is the vector $(x+1, y+1, z+1)$.

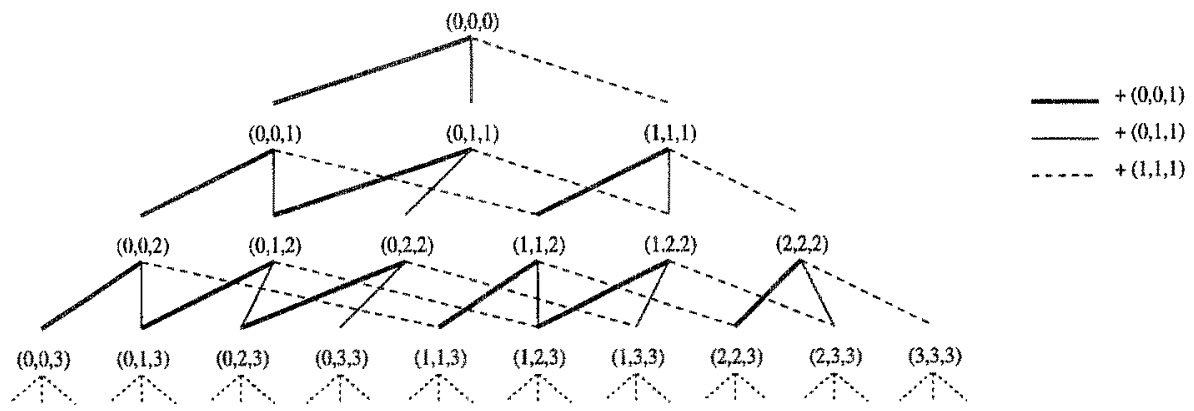

The development of grammar for $3 \mathrm{D}$ objects recognition is not recent. P.S.P. Wang [Wan89][SNW 92][Wan92] had introduced a 3D array grammar to construct $3 \mathrm{D}$ objects using the notion of neighbourhood and adjancy. Here the study is limited to naive planes and we limit neighbourhood to tricubes. The grammar we propose is in the same time a geometric way to recognize naive planes but also an analytical model because it is linked to normal vector of naive planes.

Looking at the tricubes'table of Debled[DR95], we have for each tricube the normal vector $(a, b, c)$ containing this tricube and such as $\mathrm{c}$ is minimal. We can remark that the 40 tricubes involve only 18 different normal vectors. Using the tree, we decompose these 18 vectors in a sum of 2 vectors $\left(u_{i}, v_{i}, w_{i}\right)$ with $u_{i} \wedge v_{i} \wedge w_{i}=1$. So we have the following table (at the right of the arrow we 
have the normal vector and its decomposition and at the left of the arrow, we have the list of tricubes which compose the plane):

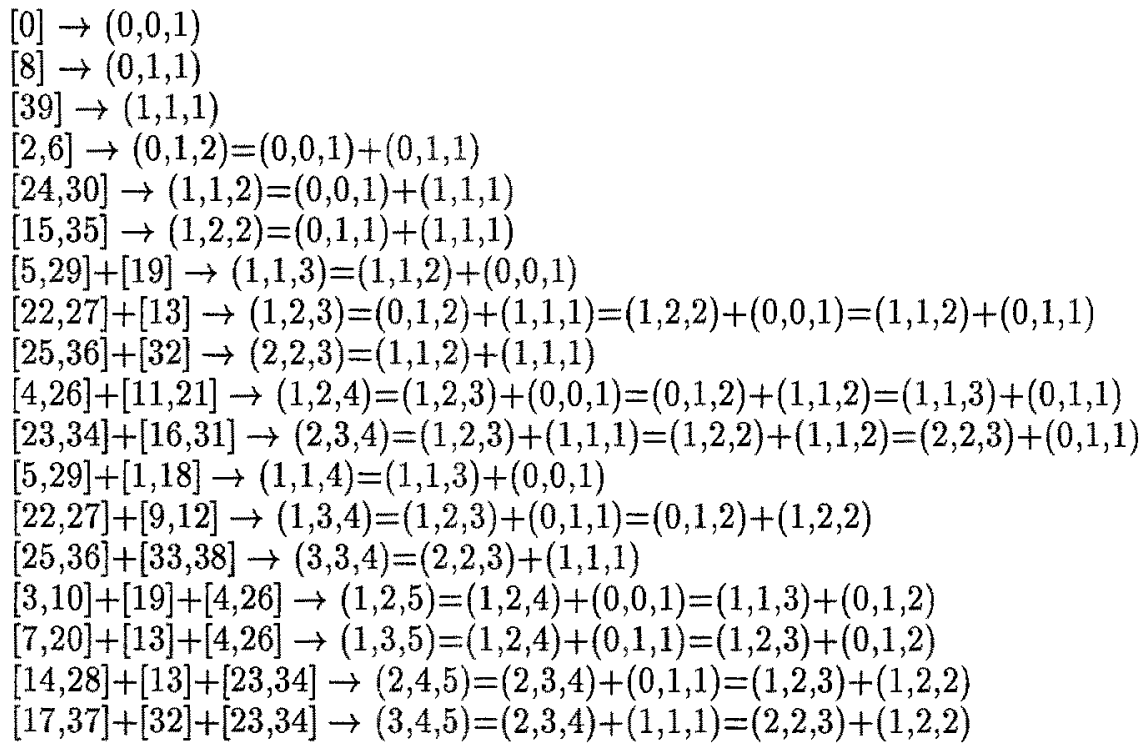

From these formulae, we extract the following grammar:

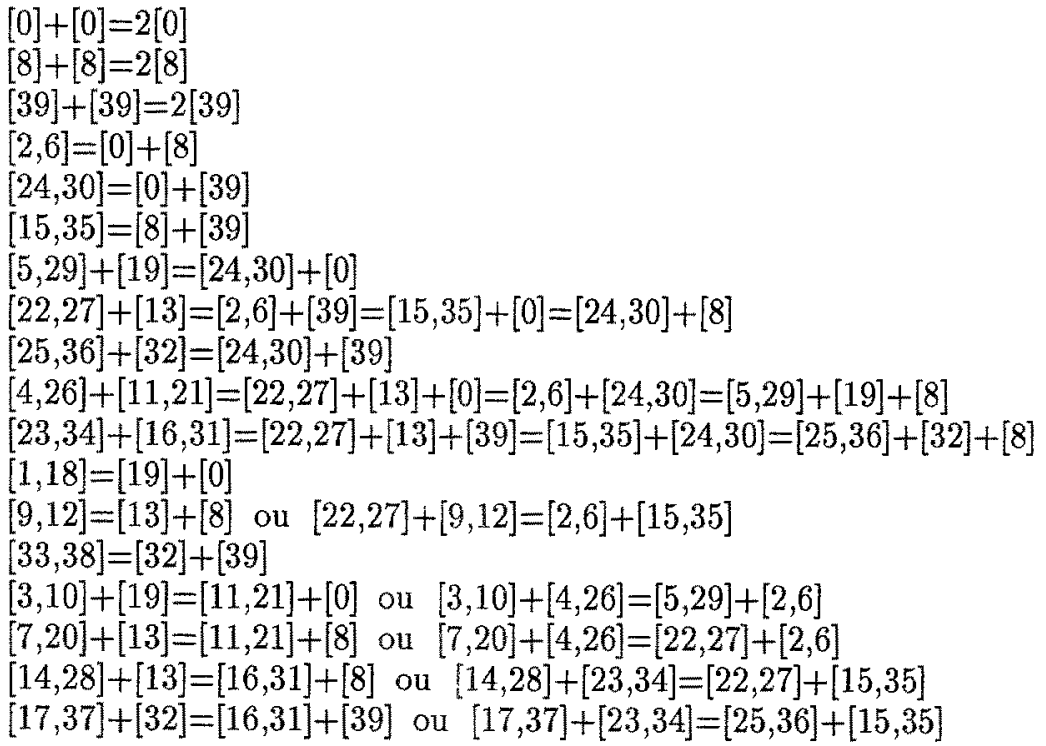

Let $P$ be a naive plane with normal vector $(a, b, c)$ with the conditions $0 \leq$ $a \leq b \leq c$. Such a plane is represented by the point $\left(\frac{a}{c}, \frac{b}{c}\right)$ corresponding to the intersection of its normal vector with plane $(z=1)$. This point belongs to the triangle of vertices $A(0,0), B(0,1)$ and $C(1,1)$ respectively associated to normal vectors $(0,0,1),(0,1,1)$ and $(1,1,1)$. 
Definition 2. Let $P(a, b, c, \mu)$ be a naive plane identified by its tricubes $\left(T_{1}, T_{2}, \ldots, T_{n}\right)$, $n \leq 9$. We suppose $a \wedge b \wedge c=1$ and $0 \leq a \leq b \leq c$.

$P$ is an exact plane if it is the unique plane containing the tricubes $\left(T_{1}, T_{2}, \ldots, T_{n}\right)$.

Example. The planes of normal vectors $(0,0,1),(0,1,1)$ and $(1,1,1)$ contain respectively only one tricube [0], [8] and [39]. They are exact planes.

We propose the following algorithm to construct exact planes. It is based on "barycentric construction" of rationnal points.

Let $P_{1}$ be an exact plane identified by its normal vector $\left(a_{1}, b_{1}, c_{1}\right)$ and its $n_{1}$ tricubes $\left(T_{1}, T_{2}, \ldots, T_{n_{1}}\right)$.

Let $P_{2}$ be an exact plane identified by its normal vector $\left(a_{2}, b_{2}, c_{2}\right)$ and its $n_{2}$ tricubes $\left(T_{1}^{\prime}, T_{2}^{\prime}, \ldots, T_{n_{2}}^{\prime}\right)$.

A new exact plane $P_{3}$ can be constructed from $P_{1}$ and $P_{2}$ if there exits a rule issued from the previous grammar which associates tricubes of $P_{1}$ with tricubes of $P_{2}$. In this case, the trace of the normal vector of the plane $P_{3}$ will be equal to the gravity center between trace of normal vectors of $P_{1}$ and $P_{2}$ weighted respectively by the number of tricubes appearing in the corresponding planes.

Example 1. Let $P_{1}$ be the plane of normal vector $(0,0,1)$ generated by the single tricube $[0]$ and let $P_{2}$ be the plane of normal vector $(1,1,1)$ generated by the single tricube [39].

In figure $6(\mathrm{a}), P_{1}$ is illustrated by the point $(0,0)$ and $P_{2}$ by the point $(1,1)$. Using rule $[0]+[39]=[24,30]$, we can generate the exact plane $P_{3}$ such as the trace of its normal vector, here $(1,1,2)$, is equal to $\frac{1}{2}[(0,0)+(1,1)]=\left(\frac{1}{2}, \frac{1}{2}\right)$ and containing only tricubes $[24,30]$ (see fig.6(b)).

The correspondance between $(1,1,2)$ and $[24,30]$ is issued from the consultation of the first table (see also Annexe).

Example 2. Let $P_{1}$ be the plane of normal vector $(0,0,1)$ generated by the single tricube $[0]$ and let $P_{2}$ be the plane of normal vector $(1,1,2)$ generated by tricubes $[24,30]$.

In figure $6(\mathrm{~b}), P_{1}$ is illustrated by the point $(0,0)$ and $P_{2}$ by the point $\left(\frac{1}{2}, \frac{1}{2}\right)$. Using rule $[24,30]+[0]=[5,29]+[19]$, we can generate the exact plane $P_{3}$ such as the trace of its normal vector $(1,1,3)$ is equal to $\frac{1}{2+1}\left[(0,0)+\left(\frac{1}{2}, \frac{1}{2}\right)\right]=\left(\frac{1}{3}, \frac{1}{3}\right)$ and containing tricubes $[5,29][19]$ (see fig.6(c)).

The correspondance between $(1,1,3)$ and $5,29,19$ is issued from the consultation of table 1 (see Annexe).

We can note in figure 6(c) that tricubes [8] and $[2,6]$ can't be associated to generate an exact plane because there is no rule between $[8]$ and $[2,6]$.

Using such algorithm we generate all the exact planes (see fig.6). 


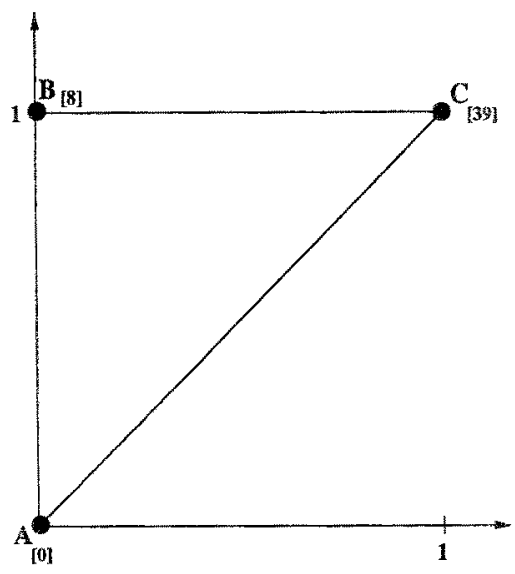

(a)
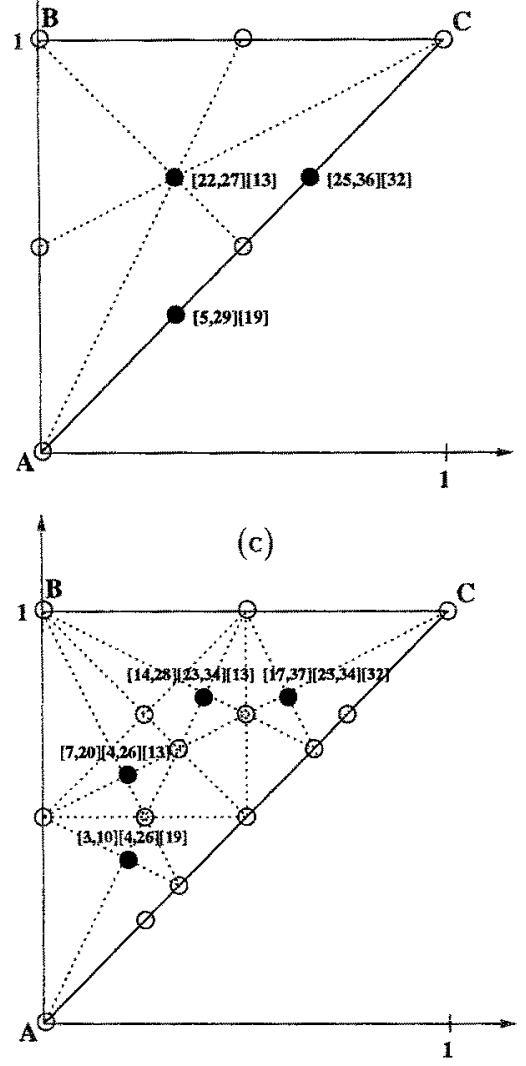

(e)

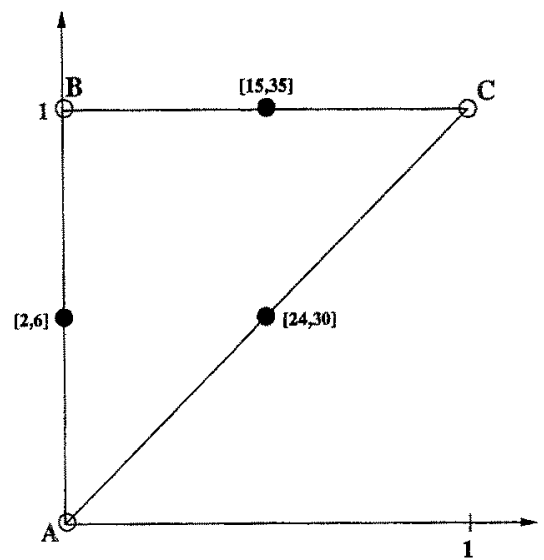

(b)

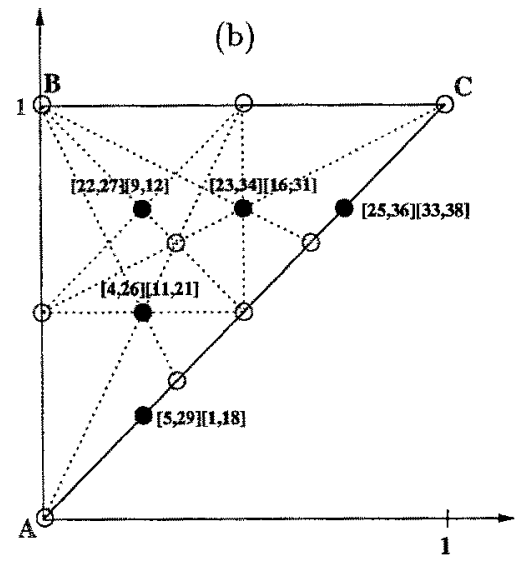

(d)

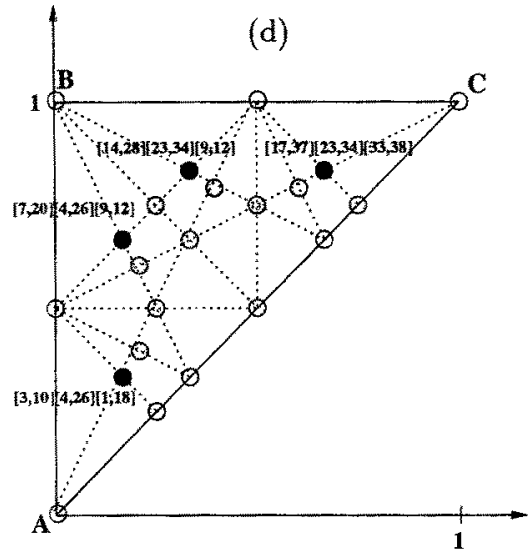

(f)

Fig. 6. Construction of the projection on plane $(z=1)$ of the normal vectors of the exact planes containing $1(\mathrm{a}), 2(\mathrm{~b}), 3(\mathrm{c}), 4(\mathrm{~d}), 5(\mathrm{e})$ and $6(\mathrm{f})$ tricubes. 
Finally, we have the following figure:

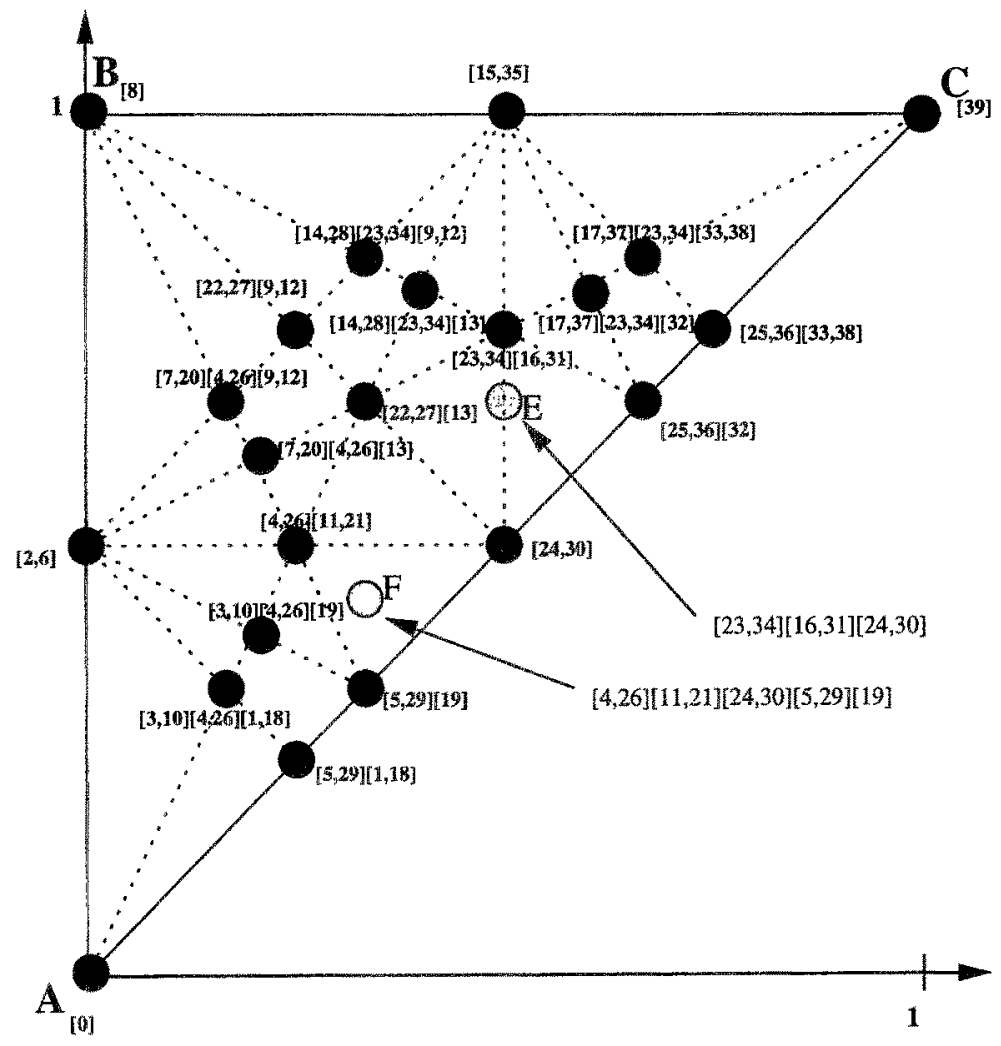

In the above figure, in addition to the 22 "exact" points we have 49 edges connecting two "exact" points and 28 polygonal areas with "exact" points as vertices. The total corresponds to the 99 possible associations of tricubes [Sch97].

\section{From normal vectors to tricubes}

To find the tricubes involved in the description of a naive plane of normal vector $(a, b, c)$ with $0 \leq a \leq b \leq c$, we can locate the trace $\left(\frac{a}{c}, \frac{b}{c}\right)$ of the normal vector in the previous figure.

Example 1. The point $E\left(\frac{3}{6} \cdot \frac{4}{6}\right)$ in the figure is supported by edge connecting planes $P_{[24,30]}$ of normal vector $(1,1,2)$ and $P_{[23,34][16,31]}$ of normal vector $(2,3,4)$. So the plane of normal vector $(3,4,6)$ is composed with tricubes $[24,30][23,34][16,31]$.

Example 2. The point $F\left(\frac{3}{9} \cdot \frac{4}{9}\right)$ in the figure is in the triangular area which vertices are planes $P_{[24,30]}$ of normal vector $(1,1,2), P_{[4,26][11,21]}$ of normal vector 
$(1,2,4)$ and $P_{[5,29][19]}$ of normal vector $(1,1,3)$. So the plane of normal vector $(3,4,9)$ is composed with tricubes $[24,30][4,26][11,21][5,29][19]$.

Here we propose an algorithm which takes into account the position of the trace into the triangle $\mathrm{ABC}$. This position will be compared with positions of tricubes $[0],[8],[39],[24,30],[2,6]$ and $[15,35]$.

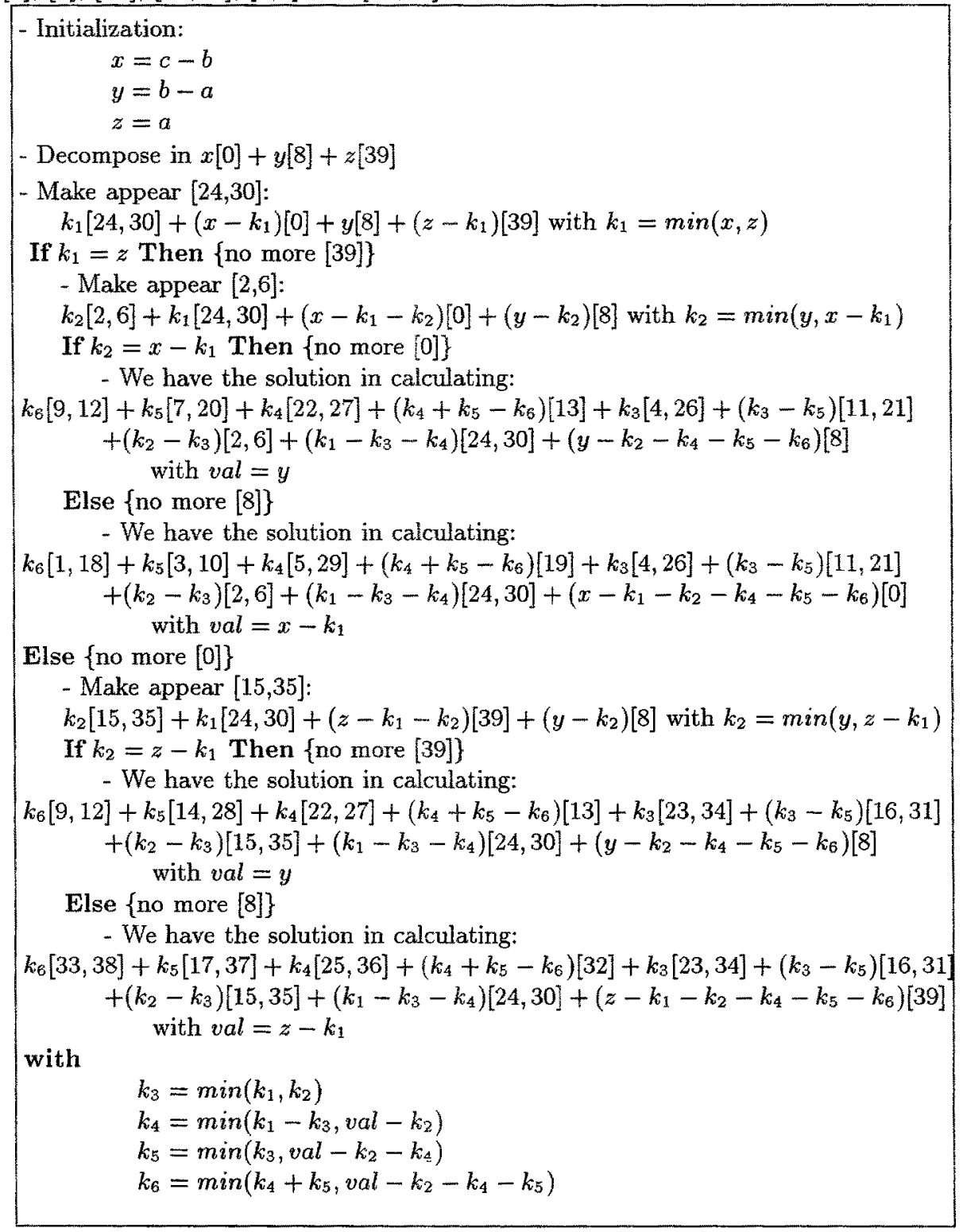

Example. If we examine the normal vector $(3,5,8)$. This vector can be decomposed as $3(0,0,1)+2(0,1,1)+3(1,1,1)$. So we have $3[0]+2[8]+3[39]$. With the 
algorithm, we obtain $[24,30]+2[22,27]+2[13]$. The plane $\mathrm{P}(3,5,8,0)$ is formed with tricubes 22,27,13, 24 and 30 . Figure 7 illustrates the configurations of tricubes around a leaning point of this same plane (see property 3 ).

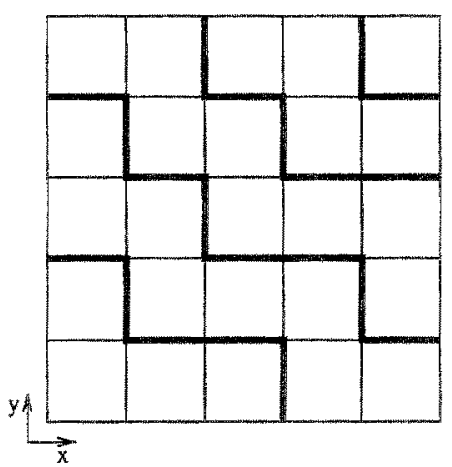

Projection of voxels which are around a lower leaning point.
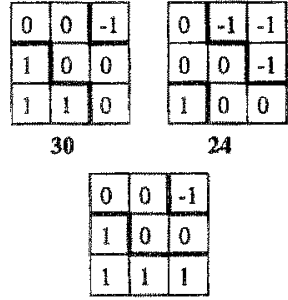

27

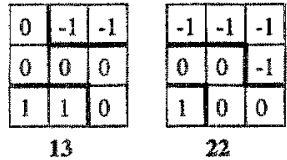

Projection of voxels appearing in the plane.

Fig. 7. Configurations of tricubes appearing in plane $P(3,5,8,0)$.

\section{Conclusion}

This work describes the way to associate normal vector of a plane and basic elements (tricubes). From a list of tricubes appearing in a set of voxels near leaning points, we are able to say if these tricubes can coexist in a naive plane. A geometric model to recognize naive digital plane has been presented and we retrieve results issued from other analytic method [Sch97].

In perspective we are working on the polyhedrization of a set of voxels and on relations between our coding of tricubes and the umbrella graph presented by Françon [Fra95].

\section{References}

[BF94] Ph. Borianne and J. Françon. Reversible polyhedrization of discrete volumes. In Proc. DCGI'4, pages 157-168, Grenoble,France, September 1994.

[CM91] J.M. Chassery and A. Montanvert. Géométrie discrète en analyse d'images, page 456. Hermès, Paris, 1991.

[DR95] I. Debled-Renesson. Etude et reconnaissance des droites et plans discrets. $\mathrm{PhD}$ thesis, University Louis Pasteur, Strasbourg,France, 1995.

[DRR94] 1. Debled-Renesson and J.P. Reveillès. An incremental algorithm for digital plane recognition. In Proc. DCGI'4, pages 207-222, Grenoble,France, September 1994.

[Fra95] J. Françon. Arithmetic planes and combinatorial manifolds. In Proc. DCGI'5, pages 209-217, Clermont-Ferrand,France, September 1995. 
[Fra96a] J. Françon. On recent trends in discrete geometry in computer science. In Lecture Notes in Computer Science, volume 1176, pages 3-16. S. Miguet, A. Montanvert and S. Ubéda Eds, Springer, 1996.

[Fra96b] J. Françon. Sur la topologie d'un plan arithmétique. In Theorical Computer Science, volume 156, pages 159-176. Elsevier, 1996.

[FST96] J. Françon, J.M. Schramm, and M. Tajine. Recognizing arithmetic straight lines and planes. In Lecture Notes in Computer Science, volume 1176, pages 141-150. S. Miguet, A. Montanvert and S. Ubéda Eds, Springer, 1996.

[Rev95] J.P. Reveillès. Combinatorial pieces in digital lines and planes. In Vision Geometry IV, volume 2573. SPIE, 1995.

[Sch97] J.M. Schramm. Tricubes coplanaires. Technical report, University of Strasbourg, France, Fev. 1997.

[SNW92] A. Saoudi, M. Nivat, and P.S.P. Wang(Eds.). Parallel image processing. In International journal of pattern recognition and artificial intelligence, volume 6. WSP, 1992.

[Wan89] P.S.P. Wang(Ed.). Array grammars, patterns and recognizers. In International journal of pattern recognition and artificial intelligence, volume 3 . WSP, 1989.

[Wan92] P.S.P. Wang. Parallel image analysis : proceedings. In ICPIA'92, Lecture Notes in Computer Science, volume 654. A. Nakamura, M. Nivat, A. Saoudi, P.S.P. Wang and K. Inoue (Eds.), Springer, 1992.

\section{Annexe: The 40 configurations of tricubes}

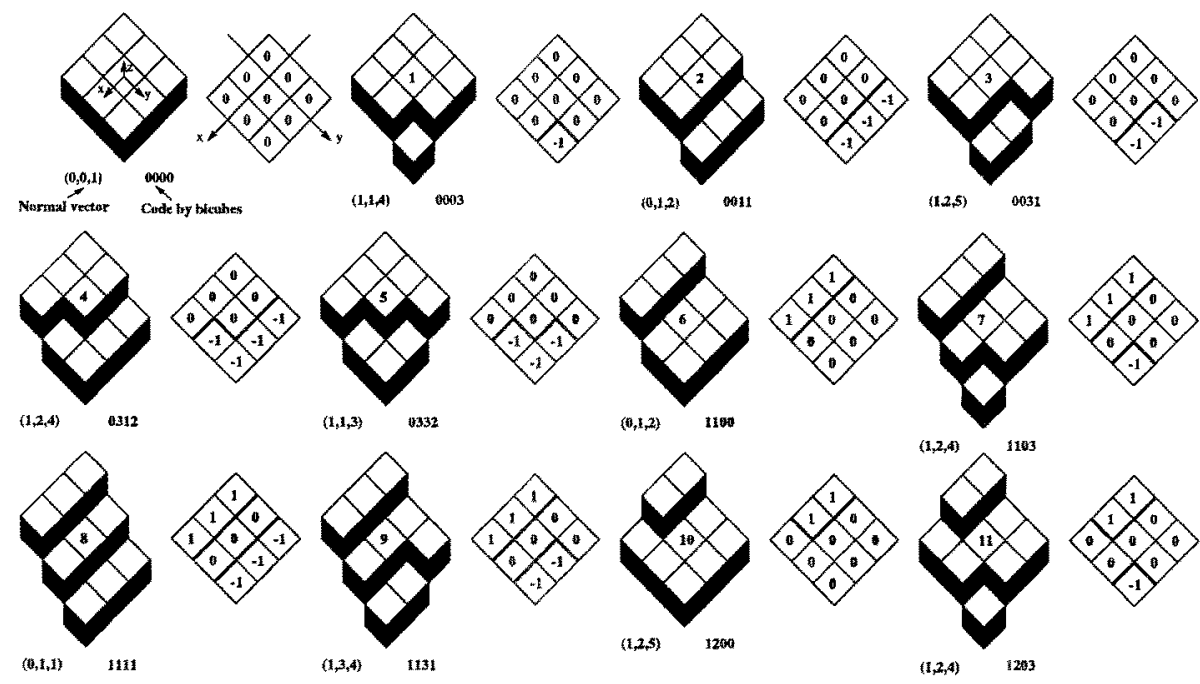




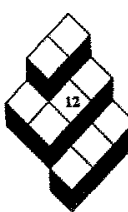
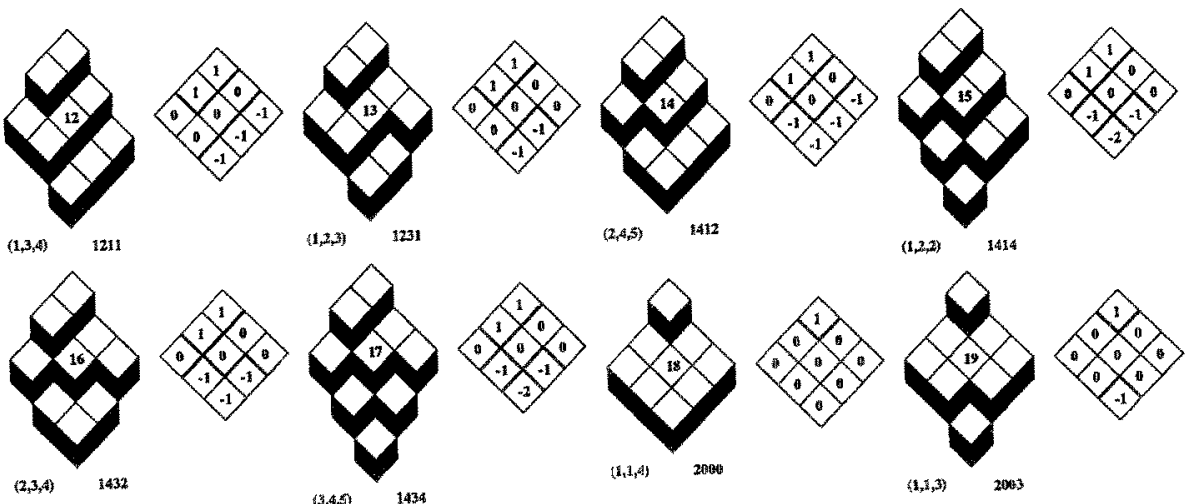

(20)
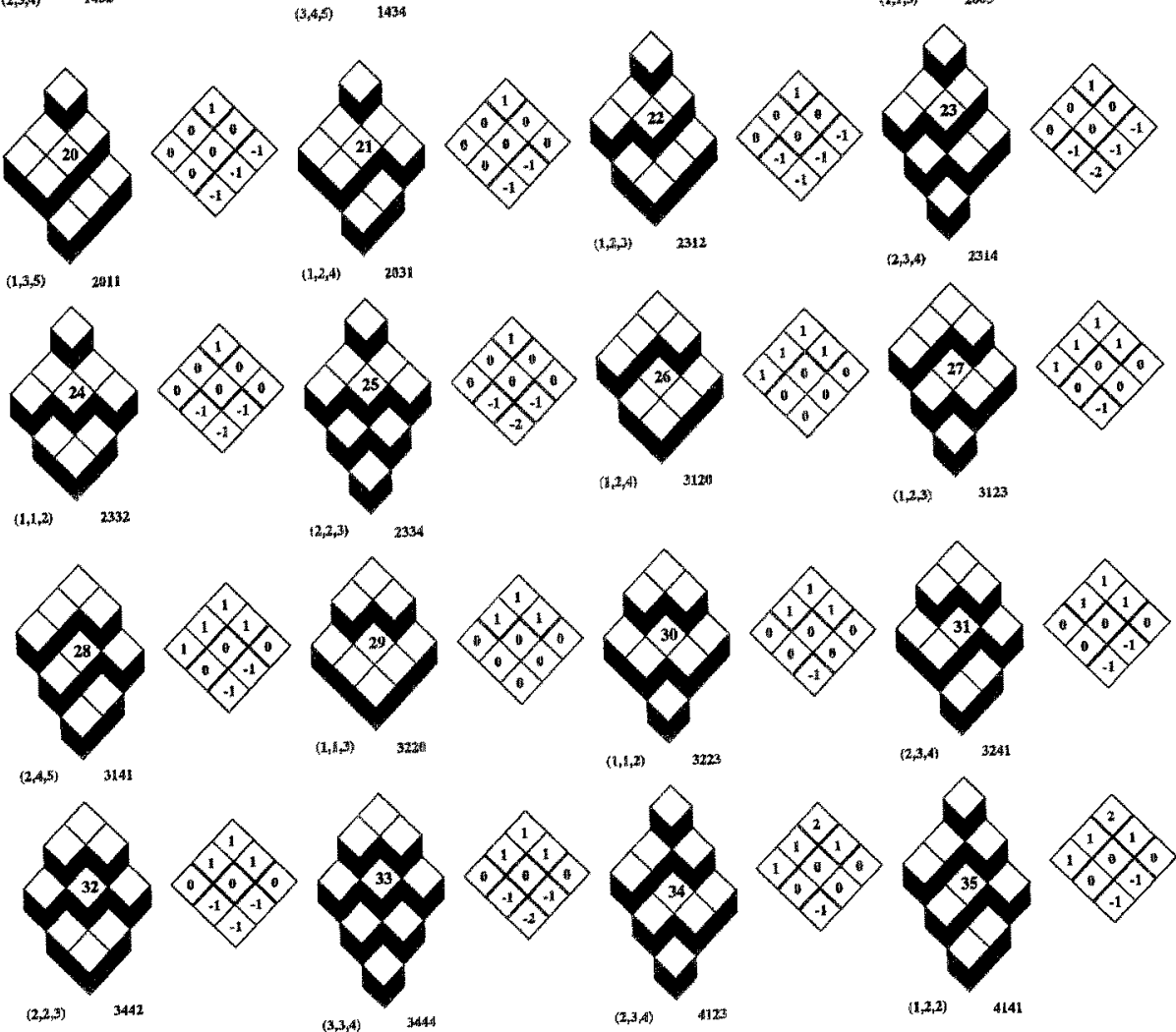

$\{2,3,4) \quad 3241$
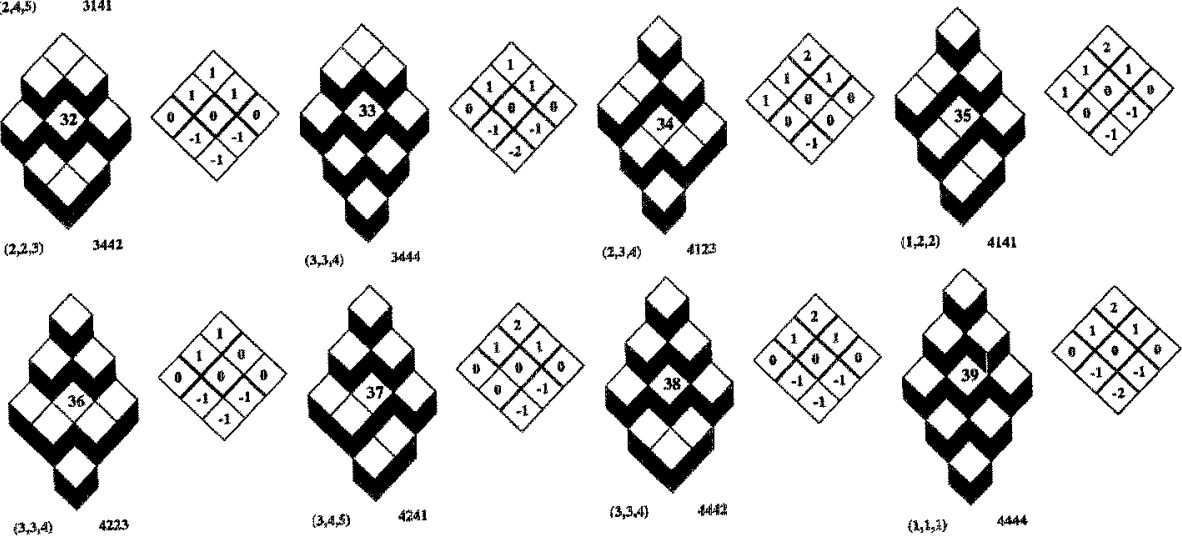